DOI: https://doi.org/10.17816/clinutr60533

Контроль гликемии у пациентов в отделении нейрореанимации

К.Ю. Крылов ${ }^{1,2}$, И.А. Савин ${ }^{2}$, С.В. Свиридов ${ }^{1}$, И.В. Веденина ${ }^{1}$, М.В. Петрова ${ }^{3,4}$, А.Н. Воробьев ${ }^{3}$, М. Рубанес ${ }^{4}$

${ }^{1}$ Российский национальный исследовательский медицинский университет имени Н.И. Пирогова, Москва, Российская Федерация

${ }^{2}$ Национальный медицинский исследовательский центр нейрохирургии имени академика Н.Н. Бурденко, Москва, Российская Федерация

${ }^{3}$ Федеральный научно-клинический центр реаниматологии и реабилитологии, Москва, Российская Федерация

${ }^{4}$ Российский университет дружбы народов, Москва, Российская Федерация

\begin{abstract}
У пациентов в критическом состоянии часто развивается гипергликемия как следствие метаболического ответа на травму и стресс. В ответ на любое тяжелое повреждение организм пациента реагирует увеличением производства собственной глюкозы и, следовательно, развитием гипергликемии, т. е. наблюдается адаптационная реакция, направленная на скорейшее восстановление повреждений. Таким образом, глюкоза является незаменимым субстратом в критическом состоянии для обеспечения репарационных процессов. Выраженная и стойкая гипергликемия связана с неблагоприятными исходами и считается независимым предиктором госпитальной смертности. Остается предметом споров, является ли гипергликемия просто маркером величины стрессовой реакции и, таким образом, суррогатным показателем тяжести заболевания или же она является причиной неблагоприятных исходов. Несколько лет назад были опубликованы исследования, которые показывали, что поддержание гликемии строго в пределах нормальных значений улучшает результаты лечения. Таким образом, отчетливо прослеживается эволюция взглядов исследователей на эту тему, и в настоящий момент времени дискуссии в научной литературе не ослабевают. В то же время вопрос о том, какой уровень глюкозы следует поддерживать у пациентов в нейрореанимации, всегда оставался спорным. В настоящем обзоре литературы авторы проанализировали современные рекомендации по лечению пациентов с нейрохирургической и неврологической патологией в критическом состоянии на предмет контроля гликемии.
\end{abstract}

Ключевые слова: контроль глюкозы; нейрореанимация; гипергликемия; гипогликемия; субарахноидальное кровоизлияние; черепно-мозговая травма; внутричерепное кровоизлияние.

Для цитирования: Крылов К.Ю., Савин И.А., Свиридов С.В., Веденина И.В., Петрова М.В., Воробьев А.Н., Рубанес М. Контроль гликемии у пациентов в отделении нейрореанимации // Клиническое питание и метаболизм. 2020;1(4):190-196. DOI: https://doi.org/10.17816/clinutr60533

Поступила: 10.02.2021 Принята: 15.06.2021 Опубликована: 18.06.2021

\title{
Glycemic Control in Neurological Intensive Care Unit Patients
}

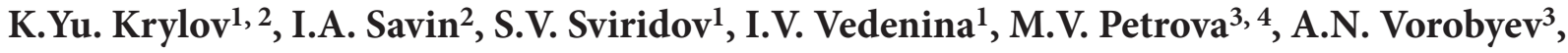 M. Rubanes ${ }^{4}$}

${ }^{1}$ The Russian National Research Medical University named after N.I. Pirogov, Moscow, Russian Federation

${ }^{2}$ N.N. Burdenko National Medical Research Center of Neurosurgery of the Ministry of Healthcare of the Russian Federation, Moscow, Russian Federation

${ }^{3}$ Federal Research and Clinical Center of Intensive Care Medicine and Rehabilitology, Moscow, Russian Federation

${ }^{4}$ Peoples' Friendship University of Russia (RUDN University), Moscow, Russian Federation

Critically ill patients often develop hyperglycemia because of the metabolic response to trauma and stress. In response to any form of damage to the organism, it reacts by increasing its own glucose production which subsequently causes hyperglycemia. This adaptive reaction of the organism is directed to aid in the rapid restoration after the damage. Therefore, glucose is an indispensable substrate in the critically ill which aids the reparation process. Severe and persistent hyperglycemia is associated with unfavorable outcomes and is considered to be an independent predictor of in-hospital 
mortality. The discussion remains on whether hyperglycemia is just a marker of increased stress which makes it a surrogate indicator of disease severity or if it is the reason for the unfavorable outcome. A few years ago, several published articles suggested that a «tight» glycemic control within the normal range improves treatment outcome. Over time, researchers have changed their point of view and currently there is a discussion on this matter in the scientific literatures. At the same time, the question of what glycemic level should be maintained for patients in the Neurological Intensive Care Unit is a matter of discussion. In this review, the authors analyzed the latest guidelines on treatment of critical patients with neurosurgical and neurological pathologies, specifically the glycemic control in this category of patients.

Keywords: glycemic control; neurointensive care unit; hyperglycemia; hypoglycemia; subarchnoid hemorrhage; traumatic brain injury; intracranial hemorrhage.

For citation: Krylov KY, Savin IA, Sviridov SV, Vedenina IV, Petrova MV, Vorobyev AN, Rubanes M. Glycemic Control in Neurological Intensive Care Unit Patients. Clinical nutrition and metabolism. 2020;1(4):190-196. DOI: https://doi.org/10.17816/clinutr60533

Received: 10.02.2021 Accepted: 15.06.2021 Published: 18.06.2021

\section{Введение}

Гипергликемия - одно из последствий эндокринно-метаболической реакции на тяжелый стресс и тяжелое повреждение [1]. Гипергликемия присутствует почти у всех пациентов в критическом состоянии.

\section{Причина развития гипергликемии}

\section{у пациентов в критическом состоянии}

В ответ на любое тяжелое повреждение организм пациента реагирует увеличением производства собственной глюкозы и, следовательно, развитием гипергликемии. Это адаптационная реакция организма, направленная на скорейшее восстановление повреждений. Именно поэтому глюкоза является незаменимым субстратом в критическом состоянии для обеспечения репарационных процессов. Она используется в качестве источника энергии в гипоксичных и воспаленных тканях и заживающих ранах, в которых митохондрии еще не развиты, или там, где свободные жирные кислоты не могут достичь клеток из-за отсутствия капилляров [2].

Основными потребителями глюкозы в организме являются иммунные клетки, фибробласты и грануляционная ткань, а также головной мозг. Увеличение производства глюкозы хоть и является жизненно важным процессом для выживания организма в критическом состоянии, но в данном аспекте представляет собой один из разрушающих факторов, провоцирующих развитие гипергликемии [2].

Выраженная и стойкая гипергликемия связана с неблагоприятными исходами и считается независимым предиктором госпитальной смертности [3]. Остается предметом споров, является ли гипергликемия просто маркером величины стрессовой реакции и, таким образом, суррогатным показателем тяжести заболевания или она является причиной неблагоприятных исходов $[4,5]$.

\section{Список сокращений}

САК - субарахноидальные кровоизлияния

ЧМТ - черепно-мозговая травма

\section{Инсулин как контроль гликемии \\ в отделениях интенсивной терапии}

Как следует из вышесказанного, гипергликемия, если она выраженная и длительно сохраняется в течение критического состояния, является причиной увеличения заболеваемости и смертности.

Проведено большое количество исследований, сравнивающих влияние стандартной стратегии коррекции гипергликемии и интенсивной инсулинотерапии для строгого поддержания нормогликемии на исходы критического состояния [6-14].

Так, в исследовании с участием 1548 пациентов, проведенном в хирургическом отделении интенсивной терапии в Бельгии [6], сравнивались эти два подхода. При стандартной стратегии гипергликемия считалась адаптивным механизмом, и ее коррекция инсулином начиналась только в случае, когда уровень глюкозы в крови превышал почечный порог (12 ммоль/л), выше которого появляется осмотический диурез и потенциально может возникать гиповолемия. При стратегии интенсивной инсулинотерапии поддерживался нормальный уровень глюкозы в крови натощак (4,4-6,1 ммоль/л) с использованием непрерывной инфузии инсулина. Поддержание нормогликемии с помощью стратегии интенсивной инсулинотерапии привело к снижению заболеваемости и смертности, при этом ключевую роль играли профилактика дисфункции жизненно важных органов и устранение угрозы 
развития новых тяжелых инфекций. Благоприятные эффекты вмешательства были объяснены предотвращением токсичности глюкозы и митохондриального повреждения нескольких жизненно важных типов клеток, включая иммунные [7-12], а не эффектом самого инсулина [13].

Однако в противовес исследованиям, которые сообщают о благоприятном влиянии интенсивной инсулинотерапии на исходы заболевания, самое крупное рандомизированное клиническое исследование группы NICE-SUGAR (Normoglycaemia in Intensive Care Evaluation and Survival Using Glucose Algorithm Regulation) [14] показало, что достижение уровней глюкозы ниже 6 ммоль/л увеличивает, а не снижает смертность, причем избыточная смертность связана с увеличением числа смертей от сердечно-сосудистых заболеваний и 13-кратным увеличением случаев тяжелой гипогликемии.

В настоящее время наибольшую обеспокоенность вызывает именно развитие случаев гипогликемии при использовании стратегии интенсивной инсулинотерапии для строгого поддержания нормогликемии, поскольку в большинстве рандомизированных контролируемых исследований по строгому контролю гликемии также сообщалось о повышении частоты развития гипогликемии [14-17], что было связано с повышенным риском смерти у пациентов в отделении интенсивной терапии. Тем не менее результаты исследований на животных показывают, что не сама гипогликемия, а степень чрезмерной коррекции после гипогликемии и, возможно, также гликемическая вариабельность имеют повреждающее воздействие на нейроны $[18,19]$. Следовательно, необходимо стремиться к минимизации вариабельности гликемии, которая несет в себе риск нейтрализации любого потенциального благоприятного эффекта от стратегии интенсивной инсулинотерапии и поддержания нормогликемии.

\section{Особенности метаболизма глюкозы}

\section{у пациентов с первичным повреждением}

\section{головного мозга}

В результате повреждения головного мозга возникает метаболический кризис, связанный в том числе с увеличением потребности головного мозга в основном энергетическом субстрате, таком как глюкоза [20, 21].

Исследования, сравнивающие стандартный протокол коррекции гипергликемии и интенсивную инсулинотерапию для строгого поддержания нормогликемии у пациентов нейрохирургического профиля, показывают преимущества стандартной стратегии. Так, например, в обзоре, посвященном коррекции ги- пергликемии у пациентов с тяжелой черепно-мозговой травмой (ЧМТ), было показано, что жесткий гликемический контроль (80-110 мг/дл, или 4,4-6,1 ммоль/л) вызывает увеличение регионального потребления глюкозы, что приводит к метаболическому кризису в головном мозге. Жесткий гликемический контроль может лишать мозг глюкозы, необходимой ему для жизнедеятельности и восстановления [22]. Эти результаты меняют парадигму и предлагают использование стандартной стратегии гликемического контроля вместо интенсивной инсулинотерапии.

Однако следует помнить, что гипергликемия также является одним из ведущих факторов вторичного повреждения головного мозга.

Перед нейрореаниматологом стоит непростая задача обеспечить оптимальное содержание глюкозы, чтобы избежать как гипогликемии, так и гипергликемии. Эта задача усложняется еще и тем, что большое количество исследований показывает, что при нормализации уровня глюкозы в периферической крови ее содержание в ткани головного мозга снижается ниже критических значений.

В настоящее время отсутствует единое мнение относительно способов коррекции гликемии, безопасного уровня глюкозы и способов мониторинга гликемии крови. В большинстве рекомендаций по интенсивной терапии пациентов нейрохирургического профиля гликемический контроль либо не рассматривается, либо ограничен общими рекомендациями. Несмотря на высокую важность поддержания гликемии в безопасном для пациента коридоре этот вопрос часто вообще не рассматривается в контексте интенсивного мониторинга пациентов нейрореанимационного профиля.

\section{Контроль гликемии у пациентов}

\section{с тяжелой черепно-мозговой травмой}

Исследования, проведенные на пациентах в критическом состоянии без ЧМТ, показали, что поддержание гликемии на уровне 4,4-6,1 ммоль/л с помощью интенсивной инсулинотерапии может приводить к значительному улучшению исходов [6].

Однако аналогичный подход в популяции взрослых пациентов с тяжелой ЧМТ продемонстрировал, что практика строгого контроля гликемии с помощью стратегии интенсивной инсулинотерапии может иметь негативные последствия у пациентов с тяжелой ЧМТ вследствие критического ее снижения в интерстициальной жидкости головного мозга [23].

Однако при изучении результатов последних исследований в области контроля гликемии у пациентов с тяжелой ЧМТ из-за несогласованности их показателей у авторов остались вопросы, что луч- 
ше - стратегия интенсивной инсулинотерапии или традиционная стратегия контроля глюкозы. По этой причине доказательства были оценены как недостаточные, и в настоящее время нельзя дать никаких рекомендаций по контролю глюкозы у данной категории пациентов [24].

\section{Контроль гликемии у пациентов}

\section{со спонтанными внутричерепными}

\section{кровоизлияниями}

В настоящее время на основании последних исследований были сформулированы весьма абстрактные рекомендации по контролю гликемии у пациентов со спонтанными внутричерепными кровоизлияниями, такие как «следует контролировать уровень глюкозы», «следует избегать как гипергликемии, так и гипогликемии» [25].

Высокий уровень глюкозы в крови при поступлении у данной категории пациентов является независимым предиктором повышенного риска смерти и неблагоприятного исхода, независимо от наличия сахарного диабета [6, 26-29]. Использование интенсивной инсулинотерапии для строго контроля гликемии (4,4-6,1 ммоль/л) в основном у хирургических пациентов в критическом состоянии с целью улучшения клинических исходов [6] увеличило использование этой стратегии у пациентов нейрохирургического и неврологического профиля. Однако более поздние исследования продемонстрировали повышенную частоту системных и церебральных гипогликемических состояний и, возможно, даже повышенный риск смерти у пациентов, получавших стратегию интенсивной инсулинотерапии для строгого контроля гликемии (4-6,5 ммоль/л) [23, 30, 31].

В настоящее время оптимальное лечение гипергликемии у пациентов со спонтанными внутричерепными кровоизлияниями и целевой уровень глюкозы еще предстоит уточнить.

\section{Контроль гликемии у пациентов}

\section{с субарахноидалыным кровоизлиянием}

Для пациентов с аневризматическими субарахноидальными кровоизлияниями (САК) также не было сформулировано четких рекомендаций по контролю гликемии. Тщательный контроль уровня глюкозы со строгим недопущением гипогликемии может рассматриваться как часть общего проведения интенсивной терапии пациентов с САК [32].

Несмотря на то, что последние исследования, сравнивающие стандартный и интенсивный подход к коррекции гликемии у пациентов с САК, показывают, что адекватный контроль гликемии может значительно снизить риск неблагоприятного исхода у этих пациентов [33], следует помнить, что даже нормальные уровни глюкозы в сыворотке крови могут приводить к энергетическому метаболическому кризису в головном мозге с повышением отношения лактат/пируват у пациентов с тяжелыми САК [34].

Таким образом, данные последних рекомендаций показывают, что высокие концентрации глюкозы в сыворотке связаны с более низким уровнем сознания, определяемым по шкале комы Глазго при поступлении, и неблагоприятными клиническими исходами [35], но и эпизоды гипогликемии также связаны с увеличением смертности [14] и неблагоприятными исходами у неврологических пациентов в критических состояниях [30]. Исходя из этих данных, необходимо свести к минимуму риск тяжелой гипогликемии и избежать ухудшения возможного повреждения нейронов, связанных с гипергликемией, следует также тщательно контролировать уровень глюкозы у данной категории пациентов с поддержанием целевых показателей периферической крови от 8,3 до 10 ммоль/л (150 до 180 мг/дл) [36].

В таблице приведены сводные рекомендации по концентрации глюкозы у пациентов в нейрореанимации [37].

\section{Заключение}

Вопрос рекомендаций по поддержанию уровня глюкозы у пациентов нейрореанимационного профиля, как и сами показатели, которых надо придерживаться, остаются дискутабельными. При проведении коррекции гликемии следует также стремиться к минимизации вариабельности гликемии крови. С целью проведения безопасного контроля гликемии представляется целесообразным мониторинг ее содержания в ткани головного мозга с помощью микродиализного катетера, который также мониторирует и общую эффективность интенсивной терапии пациентов нейрореанимационного профиля.

\section{Дополнительная информация}

Источник финансирования. Поисково-аналитическая работа по подготовке рукописи проведена без привлечения дополнительного финансирования со стороны третьих лиц.

Funding source. This study was not supported by any external sources of funding.

Конфликт интересов. Авторы данной статьи подтвердили отсутствие конфликта интересов, о котором необходимо сообщить.

Competing interests. The authors declare that they have no competing interests. 
Таблица. Рекомендованные значения содержания глюкозы в крови для нейрореанимационных пациентов (адаптировано из [37])

Table. Recommended blood glucose values for neurocritical care patients (adapted from [37])

\begin{tabular}{|c|c|c|c|}
\hline Состояние & Организация & Год & Рекомендация \\
\hline $\begin{array}{l}\text { Черепно-мозговая } \\
\text { травма }\end{array}$ & $\begin{array}{l}\text { Фонд черепно-мозговой травмы } \\
\text { (Brain Trauma Foundation) }\end{array}$ & 2007 & Не определено \\
\hline $\begin{array}{l}\text { Повреждение } \\
\text { спинного мозга }\end{array}$ & $\begin{array}{l}\text { Американская ассоциация неврологических } \\
\text { хирургов } \\
\text { (American Association of Neurological Surgeons) }\end{array}$ & 2013 & Не определено \\
\hline $\begin{array}{l}\text { Пациенты } \\
\text { стационара }\end{array}$ & $\begin{array}{l}\text { Американская диабетическая ассоциация } \\
\text { (American Diabetes Association) }\end{array}$ & 2010 & $\begin{array}{l}7,8-10 \text { ммоль/л } \\
(140-180 \text { мг/дл) }\end{array}$ \\
\hline \multirow{3}{*}{$\begin{array}{l}\text { Ишемический } \\
\text { инсульт }\end{array}$} & $\begin{array}{l}\text { Американская сердечная ассоциация } \\
\text { (American Heart Association) }\end{array}$ & 2013 & $\begin{array}{c}\text { Не допускать снижения } \\
<3,35 \text { ммоль/л (<60 мг/дл) }\end{array}$ \\
\hline & $\begin{array}{l}\text { Американская сердечная ассоциация } \\
\text { (American Heart Association) }\end{array}$ & 2007 & 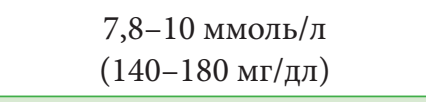 \\
\hline & $\begin{array}{l}\text { Европейская организация инсульта } \\
\text { (European Stroke Organization) }\end{array}$ & 2008 & $\begin{array}{l}<10 \text { ммоль/л } \\
(<180 \mathrm{мг/дл)}\end{array}$ \\
\hline \multirow{2}{*}{$\begin{array}{l}\text { Аневризматическое } \\
\text { субарахноидальное } \\
\text { кровоизлияние }\end{array}$} & $\begin{array}{l}\text { Американская сердечная ассоциация } \\
\text { (American Heart Association) }\end{array}$ & 2012 & $\begin{array}{c}\text { Проводить контроль } \\
\text { гликемии - не определено }\end{array}$ \\
\hline & $\begin{array}{l}\text { Европейская организация инсульта } \\
\text { (European Stroke Organization) }\end{array}$ & 2013 & $\begin{array}{l}<10 \text { ммоль/л } \\
(<180 \text { мг/дл })\end{array}$ \\
\hline \multirow{2}{*}{$\begin{array}{l}\text { Внутричерепное } \\
\text { кровоизлияние }\end{array}$} & $\begin{array}{l}\text { Американская сердечная ассоциация } \\
\text { (American Heart Association) }\end{array}$ & 2015 & $\begin{array}{c}\text { Необходим гликемический } \\
\text { контроль, нельзя допускать } \\
\text { гипер- и гипогликемии }\end{array}$ \\
\hline & $\begin{array}{l}\text { Европейская организация инсульта } \\
\text { (European Stroke Organization) }\end{array}$ & 2006 & $\begin{array}{c}<10,3 \text { ммоль/л } \\
(<185 \text { мг/дл })\end{array}$ \\
\hline
\end{tabular}

Вклад авторов. К.Ю. Крылов - планирование обзора, анализ полученных данных, рубрификация, написание обзора; И.А. Савин - планирование обзора, анализ полученных данных, редактирование обзора; С.В. Свиридов - планирование обзора, редактирование обзора; И.В. Веденина - анализ данных, редактирование; М.В. Петрова - планирование обзора, анализ полученных данных, редактирование обзора; А.Н. Воробьев - сбор данных; М. Рубанес - сбор данных, написание обзора. Все авторы подтверждают соответствие своего авторства международным критериям ICMJE (все авторы внесли существенный вклад в разработку концепции, подготовку статьи, прочли и одобрили финальную версию перед публикацией).

\section{Список литературы / Referens}

1. Cuesta JM, Singer M. The stress response and critical illness: a review. Crit Care Med. 2012;40:3283e9. doi: 10.1097/CCM.0b013e31826567eb

2. Sobotka L., ed. Basics in clinical nutrition. Galen; 2011.

3. Umpierrez GE, Isaacs SD, Bazargan N, et al. Hyperglycemia: an independent marker of in-hospital mortality in pa-
Authors' contribution. K. Y. Krylov - planning of manuscripts/study, data analysis, rubrification/planning, writing of manuscript; I. A. Savin - planning of study, data analysis, editing of manuscript; S.V. Sviridov planning of study, editing of manuscripts; I.V. Vedenina data analysis, editing of manuscript; M. V. Petrova planning of study, data analysis, editing of manuscript, A.N. Vorobev - data collection; M. Rubanes - data collection, writing of manuscript. All authors made a substantial contribution to the conception of the work, acquisition, analysis, interpretation of data for the work, drafting and revising the work, final approval of the version to be published and agree to be accountable for all aspects of the work.

tients with undiagnosed diabetes. J Clin Endocrinol Metab. 2002;87:978e82. doi: 10.1210/jcem.87.3.8341

4. Marik PE, Bellomo R. Stress hyperglycemia: an essential survival response! Crit Care. 2013;17:305. doi: 10.1186/cc12514

5. Marik PE, Egi M. Treatment thresholds for hyperglycemia in critically ill patients with and without dia- 
betes. Intensive Care Med. 2014;40:1049e51. doi: 10.1007/s00134-014-3344-2

6. Van den Berghe G, Wouters P, Weekers F, et al. Intensive insulin therapy in critically ill patients. $N$ Engl J Med. 2001;345:1359e67. doi: 10.1056/NEJMoa011300

7. Van den Berghe G. How does blood glucose control with insulin save lives in intensive care? J Clin Invest. 2004: 1187e95. doi: 10.1172/JCI23506

8. Mesotten D, Swinnen JV, Vanderhoydonc F, et al. Contribution of circulating lipids to the improved outcome of critical illness by glycemic control with intensive insulin therapy. J Clin Endocrinol Metab. 2004;219e26. doi: 10.1210/jc.2003-030760

9. Langouche L, Vanhorebeek I, Vlasselaers D, et al. Intensive insulin therapy protects the endothelium of critically ill patients. J Clin Invest. 2005;2277e86. doi: 10.1172/JCI25385

10. Van den Berghe G, Wilmer A, Milants I, et al. Intensive insulin therapy in mixed medical/surgical intensive care units: benefit versus harm. Diabetes. 2006;3151e9. doi: $10.2337 / \mathrm{db} 06-0855$

11. Vanhorebeek I, De Vos R, Mesotten D, et al. Protection of hepatocyte mitochondrial ultrastructure and function by strict blood glucose control with insulin in critically ill patients. Lancet. 2005;53e9. doi: 10.1016/S0140-6736(04)17665-4

12. Vanhorebeek I, Ellger B, De Vos R, et al. Tissue-specific glucose toxicity induces mitochondrial damage in aburn injury model of critical illness. Crit Care Med. 2009;1355e64. doi: 10.1097/CCM.0b013e31819cec17

13. Ellger B, Debaveye Y, Vanhorebeek I, et al. Survival benefits of intensive insulin therapy in critical illness: impact of maintaining normoglycemia versus glycemia-independent actions of insulin. Diabetes. 2006;55:1096e105 doi: 10.2337/diabetes.55.04.06.db05-1434

14. Finfer S, Chittock DR, Su SY, et al. NICE-SUGAR Study Investigators, Intensive versus conventional glucose control in critically ill patients. N Engl J Med. 2009;360:1283e97 doi: 10.1056/NEJMoa0810625

15. De La Rosa Gdel C, Donado JH, Restrepo AH, et al.; Grupo de Investigacion en Cuidado intensivo: GICI-HPTU. Strict glycaemic control in patients hospitalised in a mixed medical and surgical intensive care unit: a randomised clinical trial. Crit Care. 2008;12:R120. doi: 10.1186/cc7017

16. Arabi YM, Dabbagh OC, Tamim HM, et al. Intensive versus conventional insulin therapy: a randomized controlled trial in medical and surgical critically ill patients. Crit Care Med. 2008;36:3190e7. doi: 10.1097/CCM.0b013e31818f21aa

17. Brunkhorst FM, Engel C, Bloos F, et al.; German Competence Network Sepsis (SepNet). German Competence Network Sepsis (SepNet). Intensive insulin therapy and pentastarch resuscitation in severe sepsis. $N$ Engl J Med. 2008;358:125e39. doi: 10.1056/NEJMoa070716

18. Krinsley JS. Glycemic variability: a strong independent predictor of mortality in critically ill patients. Crit Care Med. 2008;36:3008e13. doi: 10.1097/CCM.0b013e31818b38d2

19. Ceriello A, Novials A, Ortega E, et al. Evidence that hyperglycemia after recovery from hypoglycemia worsens endothelial function and increases oxidative stress and inflammation in healthy control subjects and subjects with type 1 diabetes. Diabetes. 2012;61:2993e7. doi: 10.2337/db12-0224

20. Vespa PM, Nuwer MR, Nenov V, et al. Increased incidence and impact of nonconvulsive and convulsive seizures after traumatic brain injury as detected by continuous electroencephalographic monitoring. J Neurosurg. 1999;91: 750-760. doi: 10.3171/jns.1999.91.5.0750

21. Vespa PM, Miller C, McArthur D, et al. Nonconvulsive electrographic seizures after traumatic brain injury result in a delayed, prolonged increase in intracranial pressure and metabolic crisis. Crit Care Med. 2007;35:2830-2836.

22. Vespa P, McArthur D, Stein N, et al. Tight glycemic control increases metabolic distress in traumatic brain injury: A randomized controlled within-subjects trial. Crit Care Med. 2012;40(6):1-10. doi: 10.1097/CCM.0b013e31824e0fcc

23. Vespa P, Boonyaputthikul R, McArthur DL, et al. Intensive insulin therapy reduces microdialysis glucose values without altering glucose utilization or improving the lactate/pyruvate ratio after traumatic brain injury. Crit Care Med. 2006;34:850-856. doi: 10.1097/01.CCM.0000201875.12245.6F

24. Carney N, Totten AM, O'Reilly C, et al. Guidelines for the Management of Severe Traumatic Brain Injury, Fourth Edition. Neurosurgery. 2017;80(1):6-15. doi: 10.1227/NEU.0000000000001432

25. Hemphill JC, Greenberg S, Anderson C, et al.; American Heart Association. Stroke Council, Council on Cardiovascular and Stroke Nursing, and Council on Clinical Cardiology, Guidelines for the Management of Spontaneous Intracerebral Hemorrhage. Stroke. 2015;46:2032-2060. doi: 10.1161/STR.0000000000000069

26. Fogelholm R, Murros K, Rissanen A, Avikainen S. Admission blood glucose and short term survival in primary intracerebral haemorrhage: a population based study. J Neurol Neurosurg Psychiatry. 2005;76:349-353. doi: 10.1136/jnnp.2003.034819

27. Kimura K, Iguchi Y, Inoue T, et al. Hyperglycemia independently increases the risk of early death in acute spontaneous intracerebral hemorrhage. J Neurol Sci. 2007;255: 90-94. doi: 10.1016/j.jns.2007.02.005

28. Passero S, Ciacci G, Ulivelli M. The influence of diabetes and hyperglycemia on clinical course after intracerebral hemorrhage. Neurology. 2003;61:1351-1356. doi: 10.1212/01.wnl.0000094326.30791.2d

29. Stead LG, Gilmore RM, Bellolio MF, et al. Hyperglycemia as an independent predictor of worse outcome in non-diabetic patients presenting with acute ischemic stroke. Neurocrit Care. 2009;10:181-186. doi: 10.1007/s12028-008-9080-0

30. Oddo M, Schmidt JM, Carrera E, et al. Impact of tight glycemic control on cerebral glucose metabolism after severe brain injury: a microdialysis study. Crit Care Med. 2008;36:3233-3238. doi: 10.1097/CCM.0b013e31818f4026

31. Vespa PM. Intensive glycemic control in traumatic brain injury: what is the ideal glucose range? Crit Care. 2008;12:175. doi: 10.1186/cc6986

32. Connolly E, Rabinstein A, Carhuapoma JR, et al.; American Heart Association. Stroke Council, Council on Car- 
diovascular Radiology and Intervention, Council on Cardiovascular Nursing, Council on Cardiovascular Surgery and Anesthesia, and Council on Clinical Cardiology, Guidelines for the Management of Aneurysmal Subarachnoid Hemorrhage. Stroke. 2012;43:1711-1737. doi: 10.1161/STR.0b013e3182587839

33. Schlenk F, Vajkoczy P, Sarrafzadeh A. Inpatient hyperglycemia following aneurysmal subarachnoid hemorrhage: relation to cerebral metabolism and outcome. Neurocrit Care. 2009;11:56-63. doi: 10.1007/s12028-009-9222-z

34. Helbok R, Schmidt JM, Kurtz P, et al. Systemic glucose and brain energy metabolism after subarachnoid hemorrhage. Neurocrit Care. 2010;12:317-323. doi: 10.1007/s12028-009-9327-4

35. Hansen TK, Thiel S, Wouters PJ, et al. Intensive insulin therapy exerts antiinflammatory effects in critically ill patients and counteracts the adverse effect of low mannose-binding lectin levels. J Clin Endocrinol Metab. 2003; 88(3):1082-1088. doi: 10.1210/jc.2002-021478

36. Lee K. The NeuroICU Book, Second edition. McGraw Hill Education; 2017. P. 42-43.

37. Godoya DA, Behrouzc R, Napoli MD. Glucose control in acute brain injury: does it matter? Curr Opin Crit Care. 2016; 22:120-127. doi: 10.1097/MCC.0000000000000292

\section{Информация об авторах}

Крылов Кирилл Юрьевич, к.м.н.; адрес: Россия, 125047, Москва, ул. 4-я Тверская-Ямская, д. 16; ORCID: https://orcid.org/0000-0002-1807-7546; eLibrary SPIN: 9435-0854; e-mail: kkrylov@nsi.ru

Савин Иван Анатольевич, д.м.н., професcop; ORCID: https://orcid.org/0000-0003-2594-5441; eLibrary SPIN: 1342-7065; e-mail: savin@nsi.ru

Свиридов Сергей Викторович, д.м.н., профессор; ORCID: https://orcid.org/0000-0002-9976-8903; eLibrary SPIN: 4974-9195; e-mail: sergey.sviridov.59@mail.ru

Веденина Ирина Викторовна, к.м.н., доцент; ORCID: https://orcid.org/0000-0002-1232-6767; eLibrary SPIN: 6199-6980; e-mail: viv54@mail.ru

Петрова Марина Владимировна, д.м.н., професcop; ORCID: https://orcid.org/0000-0003-4272-0957; eLibrary SPIN: 9132-4190; e-mail: mail@petrovamv.ru

\section{Воробьев Алексей Николаевич;}

ORCID: https://orcid.org/0000-0003-3742-6171; eLibrary SPIN: 3253-7996; e-mail: avorobyev@fnkcrr.ru

Рубанес Мохан;

ORCID: https://orcid.org/0000-0002-5661-2706;

eLibrary SPIN: 3342-9257;

e-mail: drrubanesmohan@gmail.com

\section{Authors' Info}

Kirill Yu. Krylov, MD, Cand. Sci. (Med.); address: 125047, 4-th Tverskaya-Yamskaya st., 16, Moscow 125047, Russia; ORCID: https://orcid.org/0000-0002-1807-7546; eLibrary SPIN: 9435-0854; e-mail: kkrylov@nsi.ru

Ivan A. Savin, MD, Dr. Sci. (Med.), Professor; ORCID: https://orcid.org/0000-0003-2594-5441; eLibrary SPIN: 1342-7065; e-mail: savin@nsi.ru

Sergey V. Sviridov, MD, Dr. Sci. (Med.), Professor; ORCID: https://orcid.org/0000-0002-9976-8903; eLibrary SPIN: 4974-9195; e-mail: sergey.sviridov.59@mail.ru

Irina V. Vedenina, MD, Cand. Sci. (Med.), Assistant Professor; ORCID: https://orcid.org/0000-0002-1232-6767; eLibrary SPIN: 6199-6980; e-mail: viv54@mail.ru

Marina V. Petrova, MD, Dr. Sci. (Med.), Professor; ORCID: https://orcid.org/0000-0003-4272-0957; eLibrary SPIN: 9132-4190; e-mail: mail@petrovamv.ru

Alexey N. Vorobyev, MD;

ORCID: https://orcid.org/0000-0003-3742-6171;

eLibrary SPIN: 3253-7996; e-mail: avorobyev@fnkcrr.ru

Mohan Rubanes, MD;

ORCID: https://orcid.org/0000-0002-5661-2706;

eLibrary SPIN: 3342-9257;

e-mail: drrubanesmohan@gmail.com 